\title{
Radiological, Histopathological and Clinical Features of 4 Different Sarcomas of Maxillofacial Region
}

\author{
Ceyda Gürhan $^{1^{*}}$, Elif Şener ${ }^{2}$, Selen Bayraktaroğlu ${ }^{3}$, Başak Doğanavşargil Yakut ${ }^{4}$, Murat Sezak ${ }^{4}$ \\ ${ }^{I}$ Mugla Sttki Kocman University, Faculty of Dentistry, Department of Oral and Maxillofacial Radiology, Mugla, \\ Turkey \\ ${ }^{2}$ Ege University, Faculty of Dentistry, Department of Oral and Maxillofacial Radiology, Izmir, Turkey \\ ${ }^{3}$ Ege University Faculty of Medicine, Department of Radiology, Izmır, Turkey \\ ${ }^{4}$ Ege University Faculty of Medicine, Department of Pathology, Izmir, Turkey
}

*Corresponding Author: Ceyda Gürhan, Mugla Sttki Kocman University, Faculty of Dentistry, Department of Oral and Maxillofacial Radiology, Mugla, Turkey, Email: cydgrhn@gmail.com

\begin{abstract}
Sarcomas, although rarely seen in the maxillofacial region, are aggressive tumors. They are classified according to specific cells composing the tumor mass and have many sub-types such as osteosarcoma, fibrosarcoma, myeloid sarcoma. Although the aetiology of this pathology usually is not known, exposure to ionizated radiation or alkylating agents has been associated with an increased risk of developing bone sarcomas. Bone pain and / or swelling around the affected area are frequent symptoms of bone sarcomas. Treatment options (radical surgery, neoadjuvant tretament, chemotherapy and radiotherapy or combination of these) have varied according to stage and anotomical location of mass, age of patient and type of the pathology. The aim of our case series is to emphasize radiological, histopathological and clinic features of 4 different sarcoma cases which were reported as osteosarcoma, fibrosarcoma, myeloid sarcoma and chondroblastic osteosarcoma.
\end{abstract}

Keywords: bone sarcomas, CT, MRI, PET, radiological assessment

\section{INTRODUCTION}

Sarcomas are aggressive tumors and classified in two main groups as bone and soft tissue origin. It can occur in any bone, especially in the long bones of the extremities, but sarcomas of the maxillofacial region are rare. While sarcomas of the jaw constitute $5-10 \%$ of all sarcoma cases ${ }^{1}$, they constitute less than $1 \%$ of malignant tumors observed in the maxillofacial region ${ }^{2}$. Identifying the true incidence of these tumors is relatively difficult due to the rarity of their occurrence and difficulty in diagnosis and classification. Histopathologically having more than 50 subgroups such as osteosarcoma, chondrosarcoma, malignant fibrous histiocytoma and fibrosarcoma; causing this tumor to show variable histological and radiological features ${ }^{2,3}$. Osteosarcomas are the most common type of sarcomas for head and neck region ${ }^{4}$. Different age ranges have been reported in many studies for jaw sarcomas (JSs), which can be observed in every period of life $^{2,3,5}$. Therefore, it has been considered that age is not a determinative factor in differential diagnosis of this pathology. In addition, aetiological factors have not been fully known especially for sarcomas in maxillofacial region. Exposure to some factors such as ionizated radiation, alkylating agents has been associated with an increased risk of developing bone sarcomas ${ }^{2,4,6,7}$. Because of their highly aggressive nature, effective treatment of jaw sarcomas (JSs) have been possible only with early and accurate diagnosis ${ }^{2}$. Radiological imaging and detailed clinical examination are important factor in early diagnosis of JSs. At this point, Magnetic Resonance Imaging (MRI), Computerized Tomography (CT) and Positron Emission Tomography (PET) are preferred in not only for diagnosis of tumor but also in tumor staging and treatment planning ${ }^{3}$.

Treatment options of JSs are not basically different from sarcomas in long bone and are integrated in accordance with main cancer 
treatment protocols such as neoadjuvant chemotherapy followed by local resection with or without radiotherapy ${ }^{1}$. Neoadjuvant therapy which includes a therapeutic agents like chemotherapy, radiation therapy and hormone therapy has been recommended in order to reduce the dimensions of mass, especially for the management of high-grade sarcomas before a surgical treatment ${ }^{6}$. In fact, complete resection with negative margins in radical surgery is enough for cases in early term. Due to the complex anatomy of this region and its proximity to vital structures, it is often difficult to define the boundaries of the lesion. If there are any important anatomical structure that can be obstacle for surgical operation close to the mass region, radiotherapy and/or chemotherapy can be performed as primary treatment procedure ${ }^{1,6}$. Because sarcomas are one of the heterogeneous tumor groups and includes various sub-types; treatment strategies, mortality rate, outcome of treatment and prognosis of this pathology changes case to case $^{2}$.Therefore, treatment procedure should be case specific.

In our case-series, radiological, histopathological and clinic features of 4 different sarcoma cases reported as osteosarcoma, fibrosarcoma, myeloid sarcoma and chondroblastic osteosarcoma in our university hospital have been emphasized.

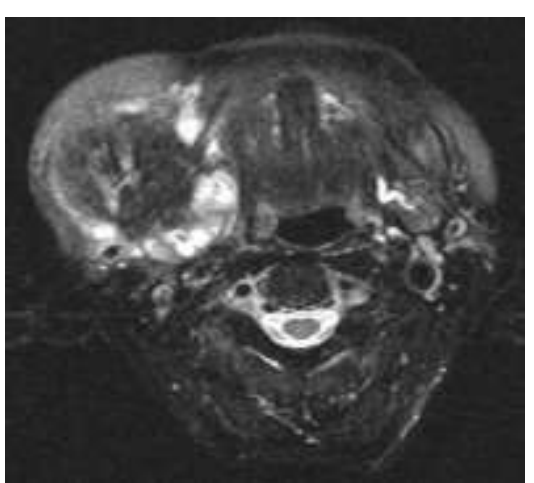

(a)

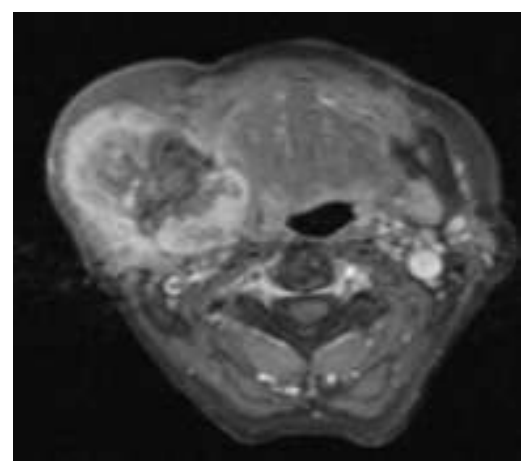

(c)

\section{Case Presentations}

2.1 Case 1: A 66-year-old female patient was referred to the out-patient clinic in faculty of dentistry for diagnosis of mass in the right mandibular retromolar region. Patient reported that swelling and pain had been present in the right side of mandible for 3-months. The medical history showed that, she had undergone cholecystectomy (17 years ago) and appendectomy (30 years ago) and under control for hypertension. After receiving informed consent, intraoral examination revealed that lesion causing extraoral swelling in right side of mandible was had extended to the soft tissue of retromolar region. The erythramatous and ulcerated clinical appearance of the lesion led to suspicion of malignancy, then the patient was consulted to the faculty of medicine for advanced diagnosis and treatment for oral lesion. In order to accurately assess the location, borders and radiological features of the pathology, further detailed radiographic analysis with Magnetic Resonance Imaging (MRI) was made. MRI images showed a large expansile destructive mass lesion with $8 \times 12 \mathrm{~cm}$ at level of right hemimandibula. Soft tissue component of mass extended into the buccal and masticator space of oral cavity. Lesion had poor defined margins (Figure 1).

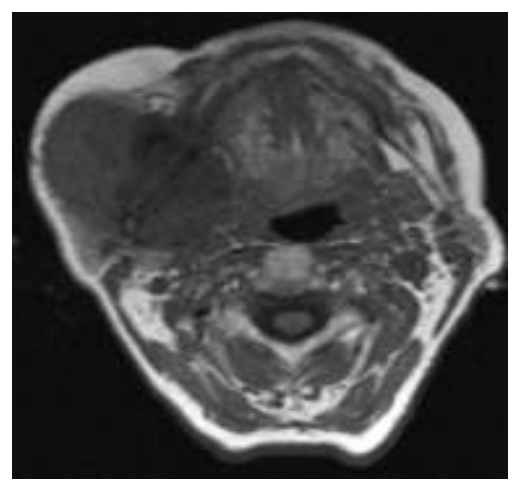

(b)

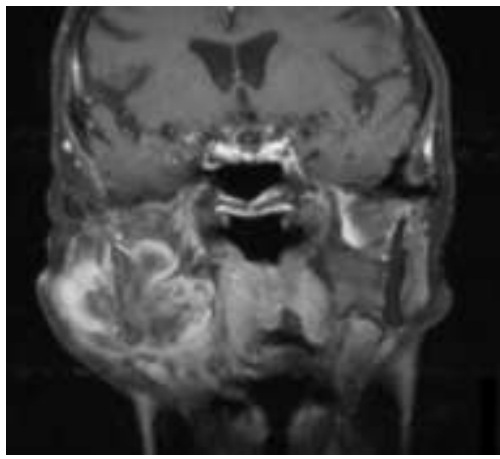

(d)

Figure1. MRI images of mandibular lesion 
On axial fat supression $\mathrm{T} 2$ weighted image and T1 weighted images lesion had central low signal intensity (Figure 1a-b). On postcontrast fat suppressed axial and coronal images, peripheral heteogenous enhancement was seen (Figure1c-d). According to the histological examination, lesion was reported as "fibrosarcoma". Treatment options of mass were discussed in head and neck malignancy council of our university. Because of lesion's wide dimensions, the neoadjuvant therapy was suggested in order to reduce size or extent of the cancer before radical treatment intervention (surgical treatment). With this aim, treatment approach was started with a radiation therapy.

2.2 Case 2: A 68-year-old male patient referred to the faculty of dentistry, department of oral and maxillofacial radiology because of the pain and swelling in the right maxilla posterior region for 3 months. Firstly, informed consent form was obtained before oral examination. He was healthy with no history of any systemic disorders. In addition to the pain and swelling, he complained of a dramatic increase in mobility of the molar teeth for 1 month. Because intraoral examination showed that gingival tissue was erythematous and hyperplastic, lesion suspected of malignancy was referred to the faculty of medicine for advanced radiological and histological examination. CT revealed an aggressive lytic lesion with $1.2 \times 1 \mathrm{~cm}$ causing destruction on the lingual bone cortex at the posterior aspect of right maxillary arch (Figure 2). The biopsy specimen sent for histopathologic examination showed that blastic cells with high mitotic index were determined in submucosal layer. Ki 67proliferation index was approximately $95 \%$. According to immunohistochemical staining [a1], the tumor cells were positive for CD 117, CD 34, Bcl-2, Bcl-6, c-myc blastic cells, while MUM1 blastic cells, CD30, CD 10, CD 20, CD 3, CD 19 were negative. CT findings combined with histopathological analysis made the definitive diagnosis of Myeloid Sarcoma and the patient was referred to the hematology-oncology service for chemotherapy.
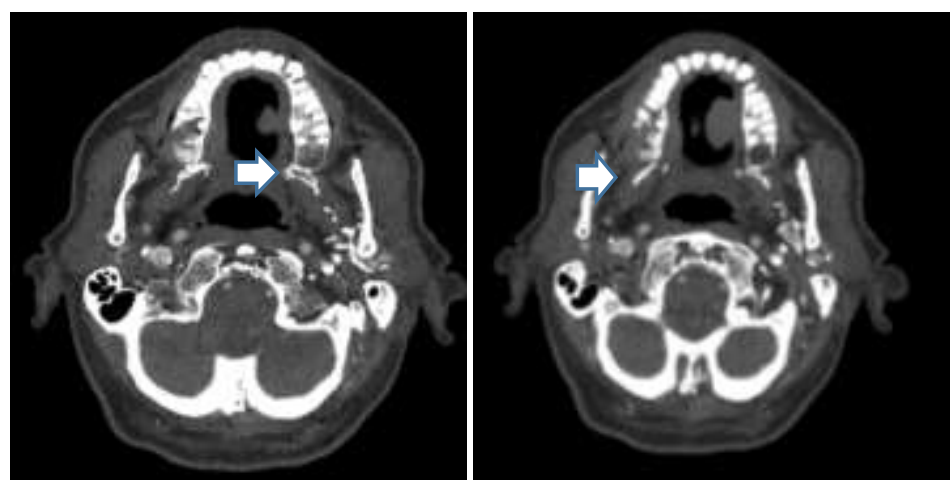

Figure2. Axial CT images of mandibular lesion. Destruction of lingual cortex and extension to adjacent soft tissue are seen (white arrow).

2.3 Case 3: A 78-year-old male patient referred to our clinic with a swelling in right posterior region of mandible 10 years ago and was diagnosed as mesenchymal mandibular tumor after radiological and histological examinations. In 2010, coronal CT scan at bone window level showed focal sclerosis and thickening at body of right mandibula (Figure 3a). A soft tissue component adjacent to the bone lesion was seen on axial CT images on soft tissue window settings (Figure $3 b$ ). After the definite diagnosis was performed, patient was treated with radiotherapy. In 2020, patient was again referred to the faculty of dentistry with the similar complaint in right region of mandible. After receiving informed consent, the ortopantomographic examination showed that wide lesion located between the right posterior region in edentulous mandibular bone and the ramus. The ground glass appearance of this lesion have masked the bone trabeculae (Figure 4). Because of the characteristic radiologic view of lesion which reminds the "sunrays " radiopacity, preliminary diagnosis was considered as osteosarcoma. Patient was consulted with doubt of osteosarcoma to the Ear, Nose \& Throat service in our university. Axial CT image at soft tissue and bone window settings showed an expansile sclerotic lesion at right hemimandibula. Lesion had led destruction at mandibular cortex and spiculated sunray periost reaction. Extension into the adjacent soft tissue was also seen (Figure 5a). 3D volume rendered CT image showed local destructive lesion at body of right mandibula (Figure $5 \mathrm{~b}$ ). On axial fat suppression T2 weighted MRI image, soft tissue component was better demonstrated (Figure 5c). Coronal contrast 
enhanced fat suppression image showed the heterogenous enhancement of soft tissue component (Figure 5d). Histopathological assessment confirmed the clinical suspicion of osteosarcoma.

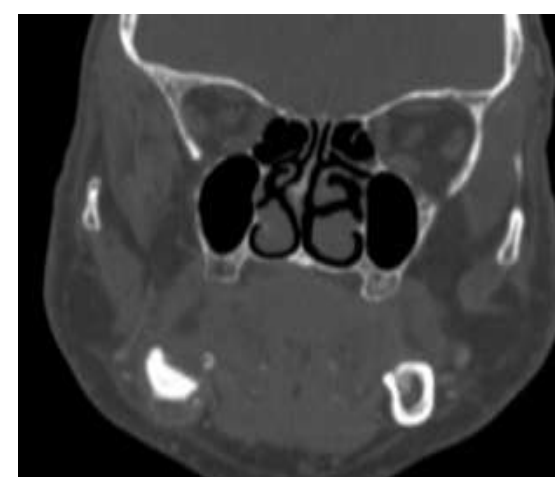

(a)

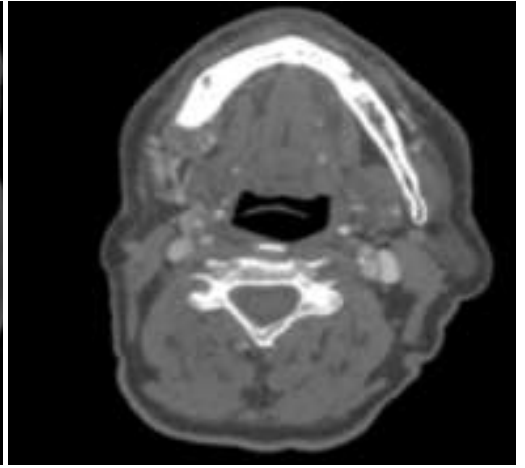

(b)

Figure3. Coronal and axial CT images of mesenchymal mandibular tumor

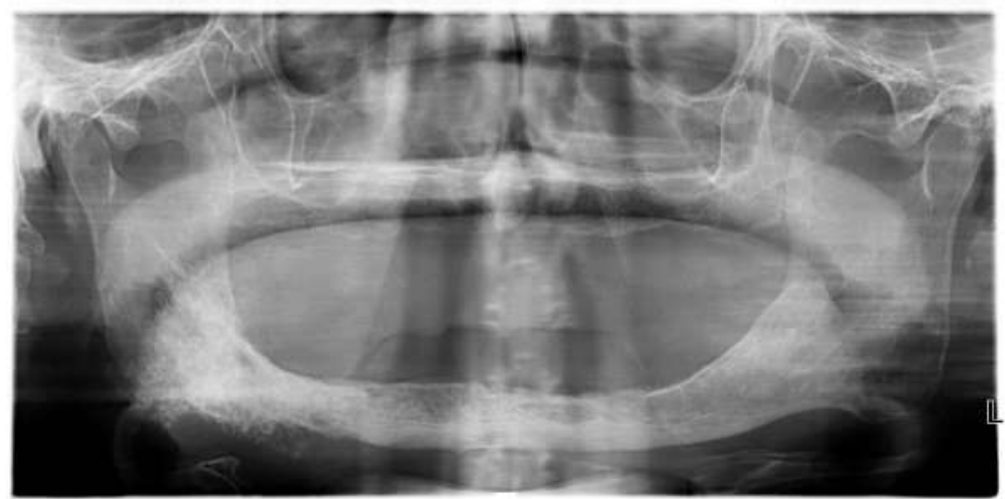

Figure4. Orthopantomograph image of mandibular lesion in right posterior region

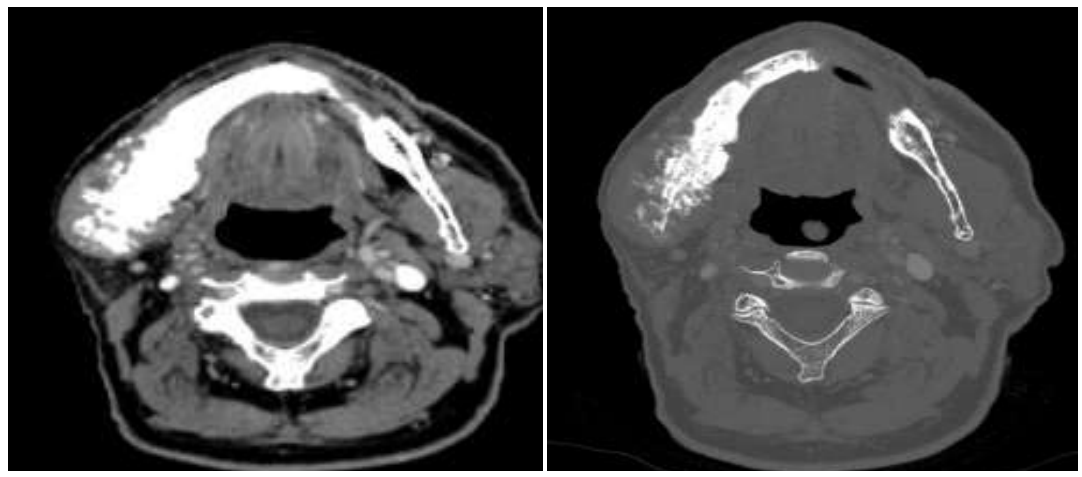

(a)

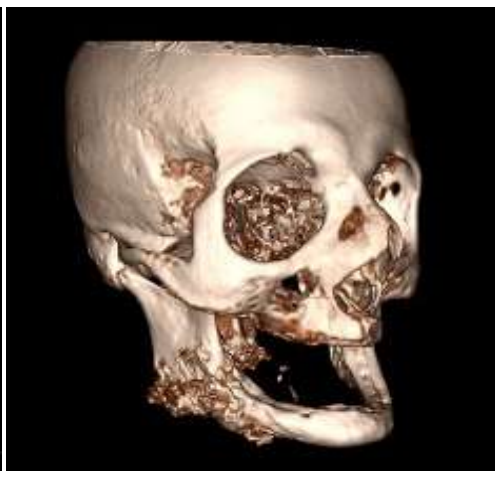

(b)

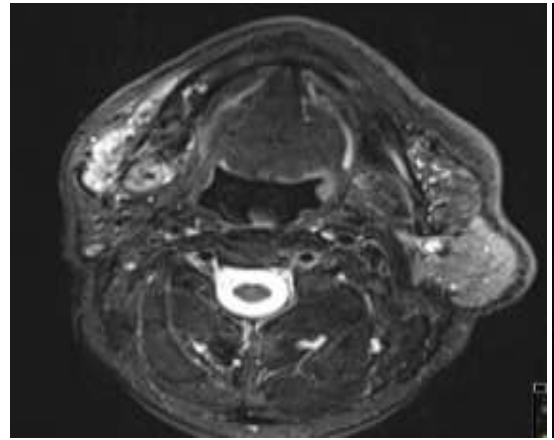

(c)

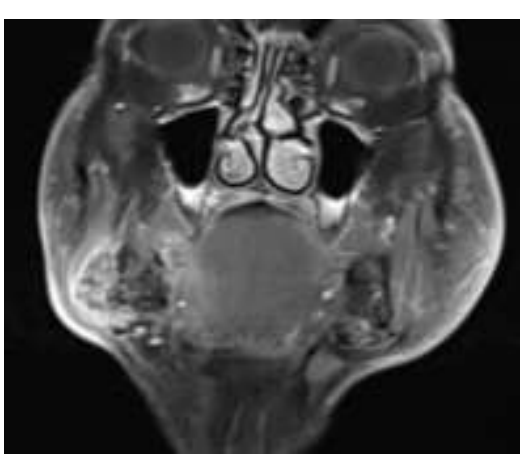

(d)

Figure5. Axial CT images $(a)$ and $3 D$ volume rendered $C T$ image of mandibular lesion $(b)$ Axial T2 weighted MRI image (c) and coronal contrast enhanced MRI image (d) of lesion 
When both recent and previous biopsy material and case's all radiologic views were comparatively evaluated, it was seen that mass had showed a slow progression and protected the majority of its histological findings during last 10 years. However, when surgical treatment was offered to patient who has a cancer recurrence in 10th years of follow-up period, he didn't approve this surgical treatment protocol.

2.4 Case 4: A 55-year old male was referred for painless swelling in left mandibular region occured 3-months ago. The patient which signed the informed consent form was consulted to the head and neck malignancy council of our university for further investigation with preliminary diagnosis of primary bone malignancy. For advanced radiologic examination, MRI and CT images were obtained in addition to the histopathological assessment. Axial CT image showed ill defined expansile lytic lesion at body of left mandibula. Lesion had led a destruction of bone cortex and extended into the surrounding soft tissue (Figure 6a). Axial T2 and contrast enhanced T1 weighted MRI images showed the extension of mass lesion to buccal space and base of oral cavity. Lesion was hyperintense on T2 weighted images and had heterogenous contrast enhacement (Figure 6b). Also, there was a compression in radix of tongue due to mass. Heterogeneous content of tumor which had an irregular border was formed from a calcified structure. Morphological features of tumor which showed a periost reaction presenting like sunray appearance confirmed the suspicious of malignant bone tumor and exact diagnosis was made with biopsy. Tumor was reported as Chondroblastic Osteosarcom. In addition, PET was obtained for cancer staging. Fused PET-CT image showed high radiotracer uptake at level of mass lesion suggesting a malignant lesion (Figure 6c).

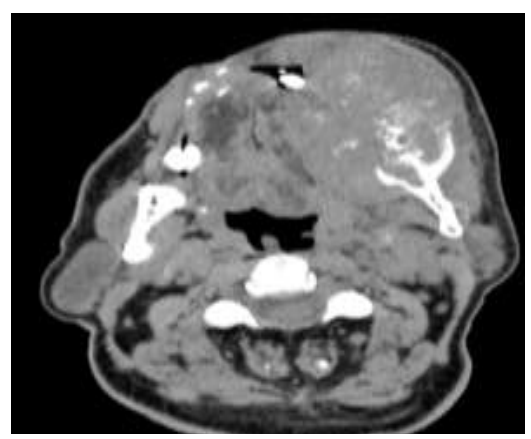

(a)
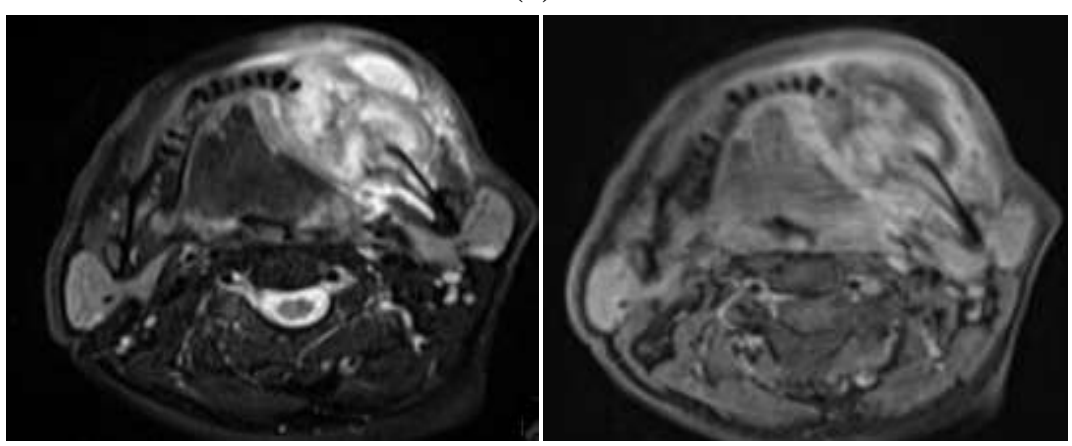

(b)

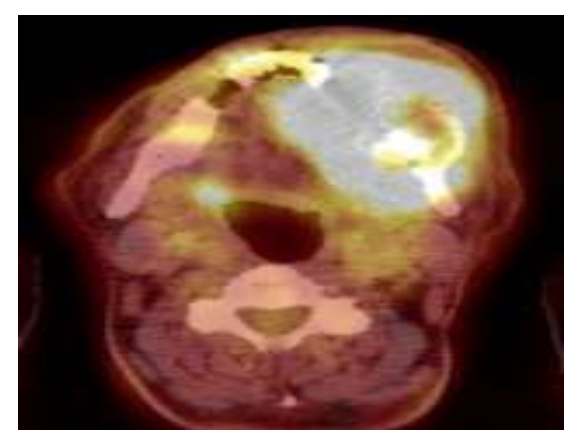

(c)

Figure6. (a) Axial CT image of mandibular lesion (b) Axial T2 and contrast enhanced T1 weighted MRI images of lesion (c) Fused PET-CT image of lesion 
According to PET images, there were the many metastatic lymph nodes in submandibular/ sublingual region which their metabolic activity cannot be evaluated due to tumor. Patient was referred for surgical treatment of primer lesion. For these suspicious lymph nodes, biopsy and ultrasonografic examination were suggested.

\section{DISCUSSION}

JSs, known as rare tumors with high grade malignancy, has a high metastasis and local recurrence rate ${ }^{6,7}$. The metastasis or recurrence rates and response to treatment varies depending on many factors such as the type of histological mass, the stage of the tumor and the treatment strategies carried out ${ }^{2}$. For example; osteosarcomas in head and neck region, known as the most common JSs, are less aggressive tumors and their prognosis are better as compared with other JSs ${ }^{2,7}$. Even, prognosis of chondroblastic type of osteosarcomas is better compared with its osteoblastic type ${ }^{3}$.

These are also important parameters affecting the survival rate of sarcomas observed in the head and neck region. Current literature has been revealed that the five-year survival rate of jaw osteosarcomas was $77 \%$ for localised disease and after complete resection ${ }^{7}$. In contrast, according to the findings of the studies evaluating the survival rate of all JS types, the survival rate of JS in five years was determined between $45 \%$ and $86 \%{ }^{2,8-10}$. Considering the possible reasons for the variability in survival rate in previous studies; it is observed that many factors such as the applied treatment methods, the size and location of the tumor, its stage, grade, subtype, and characteristics of the margin have affected the findings of presented the survival rates ${ }^{1,2}$. Another factor affecting the survival rates of JSs is the limited number of studies especially based on personal or local experiences. Because of the rarity of this pathology, there is still limited data regarding the prognosis or survival rate of these tumours ${ }^{1,7}$. For all of these reasons, it should be kept in mind that sarcomas are a large and heterogeneous group that varies in survival rate.

Considering the applied treatment approaches in the presence of this tumor, there is a consensus that the best way to control the disease is resection of the lesion as early as possible to avoid local recurrence and the need for postoperative radiotherapy ${ }^{1}$. One of the most important factors affecting the prognosis of the disease is the early diagnosis of the tumor with the help of radiological and clinical examination performed by dentists ${ }^{11}$. Usually, MRI, CT and / or combination of these imaging modalities have been preferred in the diagnosis of sarcomas due to soft and hard tissue involvement ${ }^{3}$. Other factor as important as early diagnosis of tumor is cancer staging. At this point, PET images are used in tumor staging and treatment planning. The use of the TNM Staging System and PET scans enables us to determine whether the tumor can be treated with an optimal therapeutic strategy, such as radical surgical treatment. For example, only palliative treatment can be offered for terminal cancer patients ${ }^{12}$.

JSs have presented various radiological appearance such as radiopaque, radiolucent (lytic) or mixed with or without a soft-tissue extension component and a periost reaction. However, osteosarcomas have a specific radiological view which is named as "sunray appearance" and this view have facilitated a diagnosis especially for osteosarcomas ${ }^{13}$. Except for typical appearance of osteosarcomas, there is no differential characteristic radiologic feature which separates a sarcomas from each other or from other malignant jaw lesions. Nevertheless, some radiological features such as large volume, extracompartmental extension, poorly defined margins, broad interface with underlying fascia, nonhomogeneous MRI signal intensity, high signal intensity on T2-weighted MR images and invasion of bone or neurovascular structures should be reminded a sarcomas ${ }^{14}$. Definite diagnosis in JSs should not be made with only radiologic image and should always be histopathologically and clinically confirmed. Usually, majority of cases are required the ancillary methods like immunohistochemistry and molecular diagnostic techniques for accurate diagnosis ${ }^{15}$.

When the complaints and intraoral-extraoral conditions of the patients in our case series were evaluated, it was shown that the maxillofacial swelling (with or without pain) and the expansion of the tumor to the soft tissue are common findings for all patients. These findings showed the importance of intraoral-extraoral examination performed by the dentist before referring the patient for further examinations. Dentists should remember that jaw sarcomas should be included in the differential diagnosis in cases where swelling, pain, and oral mucosal lesions are observed in the maxillofacial region.

\section{CONCLUSION}

In conclusion, sarcomas are rare but lifethreatening lesions. To diagnose the tumor in 
early stage has provided the many advantages as regards to the outcome of treatment methods and survival rates. Thus, it is important to be aware of JSs and to take them into account in the differential diagnosis of oral cavity lesions. It is expected that the case series in the present study will raise the awareness of both medical and dental practitioners about the JSs in the maxillofacial region.

\section{REFERENCES}

[1] Ketabchi A, Kalavrezos N, Newmanb L. Sarcomas of the head and neck: a 10-year retrospective of 25 patients to evaluate treatment modalities, function and survival. $\mathrm{Br}$ J Oral Maxillofac Surg. 2011;49:116-120.

[2] Vadillo RM, Contreras SJ, Canales JOG. Prognostic factors in patients with jaw sarcomas. Braz Oral Res. 2011;25(5):421-426.

[3] Kumar P, Surya V, Urs AB, Augustine J, Mohanty S, Gupta S. Sarcomas of the oral and maxillofacial region: analysis of 26 cases with emphasis on diagnostic challenges. Pathol Oncol Res. 2019;25:593-601.

[4] Kola E, Berdica L, Bushati T, Aga A, Guy A, Kola I, Horjeti E, Hoxha K, Nasir F and Musa J.Unusual Histological Variant of a Primary Osseous Tumor of the Jaw in a 27-Year-Old Female: Review of the Literature and Case Presentation. ASMS. 2020;4(5):45-48.

[5] Yamaguchi $S$, Nagasawa $H$, Suzuki T. Sarcomas of the oral and maxillofacial region: a review of 32 cases in 25 years. Clin Oral Invest. 2004; 8:52-55.

[6] Kimura Y, Tomihara K, Tachinami H, Imaue S, Nakamori K, Fujiwara K, Suzuki K, Yasuda T, Miwa S, Nakayama E, Noguchi M. Conventional osteosarcoma of the mandible successfully treated with radical surgery and adjuvant chemotherapy after responding poorly to neoadjuvant chemotherapy: a case report. J Med Case Rep. 2017;11(1):210.

[7] Bertinab H, Gomez-Brouchetc A, Rédinib F. Osteosarcoma of the jaws: An overview of the pathophysiological mechanisms. Crit Rev Oncol. 2020;156:103126.

[8] Givol N, Buchner A, Taicher S and Kaffe I. Radiological features of osteogenic sarcoma of the jaws. A comparative study of different radiographic modalities. DMFR. 1998;27:313320.

[9] Gallamini A, Zwarthoed C and Borra A. Positron Emission Tomography (PET) in Oncology. Cancers. 2014;6:1821-1889.

[10] Reddy Kundoor VK, Chelimala R, Maloth KN, Sunitha K, Thakur M. A typical radiographic presentation of osteosarcoma arising from skull and scapula: A rare case report. J Oral Maxillofac Radiol. 2016;4:14-17.

[11] Razek AA, Huang BY. Soft tissue tumors of the head and neck: imaging-based review of the WHO classification. Radiographics. 2011;31(7):1923-1954.

[12] Wang S, Shi H, Yu Q. Osteosarcoma of the jaws: demographic and CT imaging features. DMFR. 2012;41(1):37-42.

[13] Patel SG, Meyers P, Huvos AG, Wolden S, Singh B, Shaha AR. Improved outcomes in patients with osteogenic sarcoma of the head and neck. Cancer. 2002;95(7):1495-1503.

[14] Penel N, Mallet Y, Robin YM, Fournier C, Grosjean J, Ceugnart L. Prognostic factors for adult sarcomas of head and neck. Int $\mathrm{J}$ Oral Maxillofac Surg. 2008;37(5):428-432.

[15] Fernandes R, Nikitakis NG, Pazoki A, Ord RA. Osteogenic sarcoma of the jaw: a 10-year experience. J Oral Maxillofac Surg. 2007;65(7):1286-1291.

Citation: Ceyda Gürhan, Elif Şener, Selen Bayraktaroğlu, Başak Doğanavşargil Yakut, Murat Sezak. "Radiological, Histopathological and Clinical Features of 4 Different Sarcomas of Maxillofacial Region". ARC Journal of Dental Science. 2021; 6(1):9-15. DOI: https://doi.org/10.20431/2456-0030.0601003.

Copyright: (C) 2021 Authors. This is an open-access article distributed under the terms of the Creative Commons Attribution License, which permits unrestricted use, distribution, and reproduction in any medium, provided the original author and source are credited. 